\title{
PREDICTORS OF ACADEMIC PERFORMANCE, SUCCESS AND RETENTION AMONGST UNDERGRADUATE NURSING STUDENTS: A SYSTEMATIC REVIEW
}

\author{
K. Mthimunye* \\ e-mail: katlegomthimunye@icloud.com
}

\author{
F. M. Daniels* \\ e-mail: fdaniels@uwc.ac.za \\ *School of Nursing \\ University of the Western Cape \\ Cape Town, South Africa
}

\section{ABSTRACT}

Understanding the factors that predict academic performance of nursing students is one of the major goals of universities and schools of nursing globally. The aim of this study was to determine the predictors of academic performance among undergraduate nursing students. A search was carried out using various databases and journals (Science Direct, Escohost, BioMed Central, PubMed, SAGE Journals and Directory of Open Access Journals) for the period from 2006 to 2016. Two independent reviewers assessed the methodological quality of the shortlisted studies. The results are presented in textual narrative. The 17 studies included in this review represented five countries. The results of this review highlighted that older students, female gender, English language proficiency, majority ethnic status, pre-admission qualifications, high admission GPA, high supplemental application score, high pre-admission science GPA, selecting nursing as first choice for study, participating in organised music programmes, homework completion, lecture attendance, kinaesthetic learning approach, performance in psychology modules, emotional intelligence, self-control and resilience are significant predictors of academic performance among nursing students.

Keywords: academic performance, academic success, attrition, at-risk students, retention, systematic review, undergraduate nursing students

\section{INTRODUCTION AND BACKGROUND}

Maintaining academic performance, success and retention in higher education institutions (HEIs) is a global challenge. Such challenges affect nursing education too, where the critical shortage of nurses makes the output of competent nurses crucial. Several studies have been conducted to identify predictors or indicators of academic performance as well as other factors that generally influence students' performance. Nursing education has received similar attention in this regard at both theoretical and clinical levels. It has been found that numerous 
factors can forecast a student's academic performance. These factors may be of vital importance to HEIs as well as to nurse educators in identifying students who may be at risk of unsatisfactory academic performance, which limits the students' academic potential. Identification of these factors may help to reduce the attrition rate and improve the graduate output rate, thus alleviating nursing staff shortages at national and global levels.

The World Health Organization (WHO) estimates that there is a worldwide shortage of approximately 4.3 million doctors, midwives, nurses and other healthcare professionals (World Health Organization 2010). Buerhaus, Auerbach and Staiger (2009, w657-668) reported that the demand for qualified nurse practitioners was increasing at a rate of 2-3 per cent per year. To alleviate the nursing workforce shortage, the intake of nursing students needs to increase. In 2015, it was estimated that the South African Nursing Council (SANC) registered a total of 20549 nursing students for undergraduate nursing programmes at South African universities and nursing colleges. Of the 20549 students, 3808 (18.53\%) were first admissions (first-year nursing students) and 16471 (81.47\%) were re-admissions (second-year to final-year students) (SANC 2016). Most nursing schools are dependent on specific admission criteria, based on previous studies, to select high-quality prospective students who have high potential of academic success in the nursing profession. However, the selection criteria and admission requirements of nursing students in South Africa (SA) and worldwide have come under intense scrutiny in recent years, possibly owing to growing levels of attrition and continued global shortages (Newton and Moore 2009, 273-278; McNelis et al. 2010, 188-195; Smith 2016, $215-$ 218). Some authors have claimed that contextual indicators and factors such as previous academic performance, age, gender and ethnicity are significant determining parameters of academic success in training nurses (Schmidt and MacWilliams 2011, 171-174; Jeffreys 2012; Beauvais et al. 2014, 918-923; Mthimunye, Daniels and Pedro 2015).

A more comprehensive and systematic review of the factors that predict academic performance, success and retention of undergraduate nursing students (UNs) is required to ensure appropriate ways of enhancing nursing education so as to produce a cadre of nurses who will boost human resources for health. The current article presents the findings of a systematic review based on the Nursing Universal Retention and Success (NURS) model of Jeffreys (2015).

\section{METHODS}

The review aimed to systematically locate, appraise the quality of, and synthesise academic studies proposing likely predictors of academic performance amongst UNs. The main research question addressed was: What are the predictors of academic performance and success as well as the factors that may affect the retention of UNs? Cochrane guidelines for systematic reviews 
were used to guide this review (Higgins and Green 2011). After formulating the research question, systematic and explicit methods were used to identify, select and critically evaluate the quality of relevant research reports from major educational databases.

\section{Electronic search strategy}

In addressing the predictors of academic success and retention amongst nursing students, a search was conducted in January 2017 of databases and journals such as Science Direct, Escohost (Eric, Academic Search Complete, PsycARTICLES, Education Search Complete, Health Source: Nursing/Academic Edition), BioMed Central, PubMed, SAGE Journals and Directory of Open Access Journal (DOAJ) for the period 2006 to 2016. Keywords used in the search included predictors of academic performance, predictors of academic success and nursing students. The primary researcher conducted an initial search and reviewed the titles, abstracts and articles. The initial search generated 522 articles. The next phase involved eliminating duplications, which left 444 retrieved articles. Eight additional studies were considered for possible inclusion, which were obtained from other sources and from reference lists of other articles, increasing the number of studies to 452 .

\section{Criteria for considering studies for review (inclusion criteria)}

Studies were included if they met the following criteria: (1) full-text quantitative study in English; (2) prospective and retrospective cohort studies, case-control studies and crosssectional studies; (3) UNs were included as part of the sample; (4) primary outcome of interest was predictors of academic performance, success and retention amongst B.Nurs students. Secondary outcomes included factors that influenced academic performance and success; and (5) the papers were published between 2006 and 2016. Globally, the major developments in nursing education over the last decade have been in addressing nurse shortages, improving the status of nursing education, and improving the quality of nurses produced.

\section{Study selection}

Three instruments were used to ensure rigorous screening of the research studies and to ensure that all relevant data were collected. Firstly, the Title Reading and Extraction Tool (TRET) was used to select journal articles for inclusion based on the relevance of the title. A sample of 42 studies was reached, based on title reading. Secondly, the Abstract Reading Extraction Tool (ARET) was used to assess the relevance of selected journal articles for inclusion based on the relevance of the abstract. Following title reading, the abstracts of the studies were reviewed for eligibility and a sample of 20 studies was identified and retrieved. Thirdly, the Quality Assessment Tool (QAT) was used by one of the reviewers after the retrieval where possible of full-text articles to investigate their quality. The same process was then followed by another 
reviewer to determine whether the article adequately met the criteria for inclusion in the review. Any discrepancies were adjudicated and agreement reached by consensus.

\section{Methodological quality appraisal}

A quality assessment tool for quantitative studies developed by the Effective Public Health Practice Project (EPHPP) was adapted to fit the current review (Thomas 2003). The QAT was adopted from previous studies (Armijo-Olivo et al. 2012, 12-18; Puertas, Arósquipa and Gutiérrez 2013, 351-358; Bassir et al. 2014, 98-109). This tool assesses aspects of the methodologies employed and gives scores on a Likert-type scale. Following the quality appraisal of eligible studies, three studies were excluded owing to their overall weak rating. Therefore, the final sample consisted of 17 articles which were included in the systematic review. Figure 1 outlines the process involved in the systematic review.

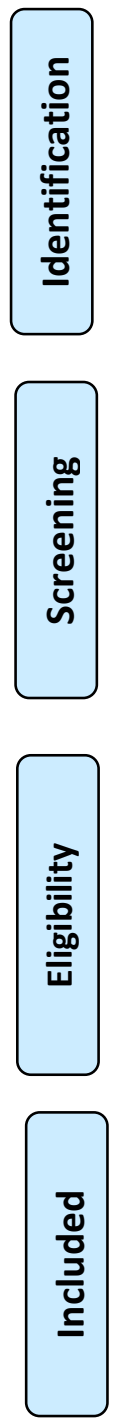

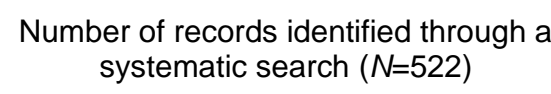
systematic search $(N=522)$

Number of additional records identified through other sources $(n=8)$

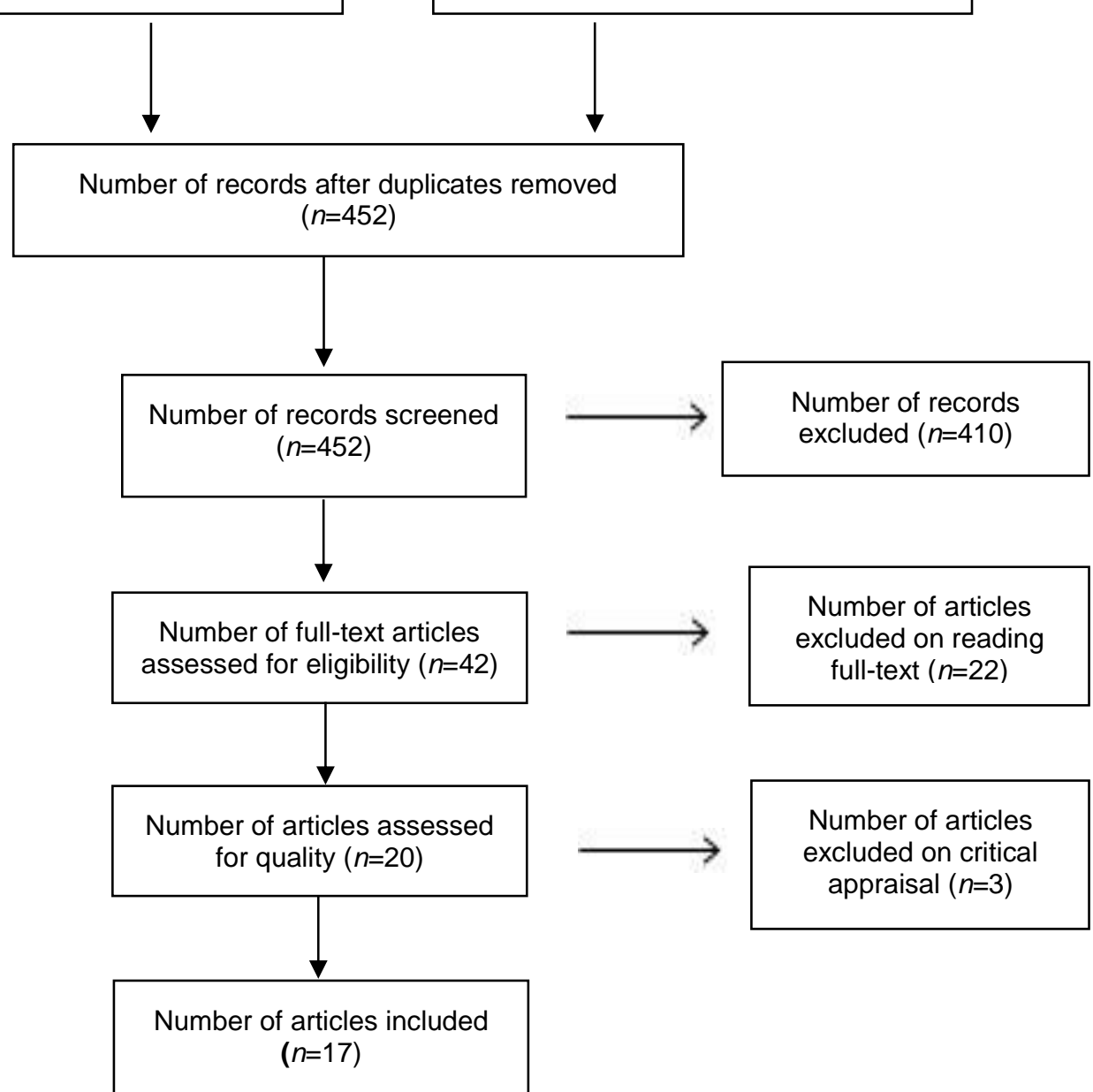

Figure 1: Flow chart of study screening process. 


\section{Data extraction and synthesis}

After completion of the methodological quality appraisal, studies that met the criteria for the categories of "strong" to "moderate" were reviewed, and a data extraction tool was used to ensure that all relevant data were extracted from the selected articles. Table 1 comprises relevant extracted information such as author and year of publication, geographical location, participants, study design, data collection instruments used and the results of the study. The studies that were included in the review measured academic performance and success (outcome variable) in various ways and at various levels of the undergraduate nursing programmes. Therefore, statistical methods (meta-analysis) were not used to analyse and summarise the results of shortlisted studies for two main reasons. Firstly, the shortlisted studies addressed heterogeneous outcome data, so it would not have been logical to cluster them together. Secondly, not all shortlisted studies provided required statistical data for possible meta-analysis. For these reasons, the results of the present review are presented in textual narrative synthesis.

\section{RESULTS}

Of the 42 studies, 20 formed part of the methodological appraisal section of the review. The criteria used for assessing the methodological quality included selection methods, study design, data collection methods, data source, withdrawals and drop-outs as well as data analyses. Of the 20 studies that formed part of the methodological appraisal section, 11 studies reached the desired outcome in the "strong" category. The 6 studies in the "moderate" category were included in the review. These studies were included because they also examined the predictive power of independent variables on the academic performance as well as academic success of nursing students. Only 3 studies fell into the "weak" category and were excluded from the study because they were rated "weak" in two or more sections of the QAT. Therefore, the remaining 17 studies that were methodologically appraised for quality were included in the final review.

Table 1: Data extraction (summary included studies)

\begin{tabular}{|c|c|c|c|c|c|}
\hline Author (year) & $\begin{array}{l}\text { Setting/ } \\
\text { location }\end{array}$ & Participants & $\begin{array}{c}\text { Study } \\
\text { design }\end{array}$ & Instrument(s) used & $\begin{array}{c}\text { Significant predictors of } \\
\text { performance }\end{array}$ \\
\hline $\begin{array}{l}\text { Cesario, } \\
\text { Cesario and } \\
\text { Cesario 2013, } \\
141-146\end{array}$ & $\begin{array}{l}\text { State of } \\
\text { Texas, } \\
\text { USA }\end{array}$ & $\begin{array}{l}\text { A total of } 309 \\
\text { undergraduate } \\
\text { nursing students } \\
\text { from } 78 \text { schools; } \\
87.4 \% \text { female, } \\
12.6 \% \text { male }\end{array}$ & $\begin{array}{l}\text { Exploratory } \\
\text { study }\end{array}$ & $\begin{array}{l}\text { Not clear. Online } \\
\text { survey } 18 \text {-item data } \\
\text { collection tool } \\
\text { (reviewed by a panel } \\
\text { of } 3 \text { expert nurse } \\
\text { educators and } 2 \\
\text { experienced music } \\
\text { educators.) }\end{array}$ & $\begin{array}{l}\text { Graduation } \\
\text { - Participating in a music } \\
\text { programme, exit GPA and } \\
\text { number of years taking } \\
\text { music lessons. } p<0.001 \text {, } \\
\text { Nagelkerke } R^{2}=0.66\end{array}$ \\
\hline $\begin{array}{l}\text { Salamonson, } \\
\text { Andrew and } \\
\text { Everett 2009, } \\
123-132\end{array}$ & Australian & $\begin{array}{l}126 \text { second-year } \\
\text { nursing students } \\
\text { (mean age } 24.8 \\
\text { years; } 89 \% \text { female, }\end{array}$ & $\begin{array}{l}\text { Prospective } \\
\text { survey }\end{array}$ & $\begin{array}{l}\text { Survey details not } \\
\text { clear. }\end{array}$ & $\begin{array}{l}\text { Homework completion } \\
(>50 \%)(\beta=0.44, p<0.001) \text {; } \\
\text { lecture attendance }(>80 \%) \\
(\beta=0.21, p=0.011) \text {; hours }\end{array}$ \\
\hline
\end{tabular}




\begin{tabular}{|c|c|c|c|c|c|}
\hline Author (year) & $\begin{array}{l}\text { Setting/ } \\
\text { location }\end{array}$ & Participants & $\begin{array}{l}\text { Study } \\
\text { design }\end{array}$ & Instrument(s) used & $\begin{array}{c}\text { Significant predictors of } \\
\text { performance }\end{array}$ \\
\hline & & $11 \%$ male) & & & $\begin{array}{l}\text { spent in part-time } \\
\text { employment }(\geq 16 \\
\text { hours/week })(\beta=-0.26, p= \\
0.001) \text { : adjusted } R^{2}=0.34\end{array}$ \\
\hline $\begin{array}{l}\text { Wan Chik et } \\
\text { al. 2012, } \\
387-393\end{array}$ & $\begin{array}{l}\text { North- } \\
\text { western } \\
\text { Peninsular } \\
\text { Malaysia }\end{array}$ & $\begin{array}{l}147 \text { first- and } \\
\text { second-year } \\
\text { nursing students } \\
\text { (mean age } 20.0 \\
\text { years; } 81 \% \text { female, } \\
19 \% \text { male) }\end{array}$ & $\begin{array}{l}\text { Prospective, } \\
\text { correlational } \\
\text { survey }\end{array}$ & $\begin{array}{l}\text { - English Language } \\
\text { Acculturation Scale } \\
\text { ELAS) } \\
\text { - Malay Language } \\
\text { Acculturation Scale } \\
\text { MLAS) } \\
\text { - Macleod Clark } \\
\text { Professional Identity } \\
\text { Scale (MCPIS-9) }\end{array}$ & $\begin{array}{l}\text { - Gender }(\beta=-0.44, \\
p<0.001) \\
\text { - Gender, age, MCPIS- } 9 \text {, } \\
\text { ELAS and MLAS (adjusted } \\
\mathrm{R}^{2}=0.181 \text { ) }\end{array}$ \\
\hline $\begin{array}{l}\text { Salamonson } \\
\text { and Andrew } \\
2006,342- \\
349\end{array}$ & $\begin{array}{l}\text { New South } \\
\text { Wales, } \\
\text { Australia }\end{array}$ & $\begin{array}{l}267 \text { second-year } \\
\text { nursing students } \\
\text { (mean age } 24.87 \\
\text { years; } 90 \% \text { female } \\
\text { and } 10 \% \text { male) }\end{array}$ & $\begin{array}{l}\text { Prospective, } \\
\text { quantitative } \\
\text { survey }\end{array}$ & $\begin{array}{l}\text { Investigator- } \\
\text { developed } \\
\text { questionnaire (used } \\
\text { in previous } \\
\text { unpublished studies) }\end{array}$ & $\begin{array}{l}\text { - Hours spent in part-time } \\
\text { employment }(p<0.001) \text {; on } \\
\text { pathophysiology }(\beta=-0.29) \text {; } \\
\text { on nursing practice ( } \beta \\
=-0.26) \\
\text { - Non-English-speaking on } \\
\text { pathophysiology }(\beta=-0.19 \text {, } \\
p=0.002) \text {; on nursing } \\
\text { practice }(\beta=-0.25 \text {, } \\
p<0.001) \\
\text { - Older age }(\geq 25 \text { years old }) \\
\text { on pathophysiology ( } \beta= \\
0.14, p=0.023) ; \text { on nursing } \\
\text { practice }(\beta=0.14, p= \\
0.036)\end{array}$ \\
\hline $\begin{array}{l}\text { Everett et al. } \\
2013,709- \\
713\end{array}$ & $\begin{array}{l}\text { Australian } \\
\text { university }\end{array}$ & $\begin{array}{l}N=730 ; \text { first-year } \\
\text { Bachelor of } \\
\text { Nursing, standard } \\
\text { entry }(n=471) \text {; first- } \\
\text { year Bachelor of } \\
\text { Nursing, graduate } \\
\text { entry }(n=259)\end{array}$ & $\begin{array}{l}\text { Prospective } \\
\text { correlational }\end{array}$ & $\begin{array}{l}\text { - ELAS } \\
\text { - Motivated } \\
\text { Strategies for } \\
\text { Learning } \\
\text { Questionnaire } \\
\text { (MSLQ) }\end{array}$ & $\begin{array}{l}\text { - Age: }>21 \text { years was found } \\
\text { to have a positive impact } \\
(p<0.001) \text { on BNSE GPA } \\
\text { - Lower levels of English- } \\
\text { language proficiency were } \\
\text { associated with poor } \\
\text { academic performance in } \\
\text { both BNSE }(p=0.001) \text { and } \\
\text { BNGE }(p<0.001) \\
\text { - Increased time spent in } \\
\text { paid work }(>16 \text { h/week) was } \\
\text { found to have a negative } \\
\text { impact on BNSE }(p=0.001) \\
\text { and BNGE }(p<0.001) \text {. }\end{array}$ \\
\hline $\begin{array}{l}\text { Glew et al. } \\
2015,1142- \\
1147\end{array}$ & $\begin{array}{l}\text { Western } \\
\text { Sydney, } \\
\text { Australia }\end{array}$ & $\begin{array}{l}2669 \text { first-year } \\
\text { nursing students } \\
\text { (mean age } 26.8 \\
\text { years; } 84.6 \% \\
\text { female and } 15.4 \% \\
\text { male; } 49.6 \% \\
\text { Australian-born, } \\
50.4 \% \text { born } \\
\text { outside of } \\
\text { Australia) }\end{array}$ & $\begin{array}{l}\text { Prospective } \\
\text { survey }\end{array}$ & $\begin{array}{l}\text { - ELAS } \\
\text { - Post-enrolment } \\
\text { English language } \\
\text { assessment (PELA): } \\
\text { designed to assess } \\
\text { student's literacy } \\
\text { skills }\end{array}$ & $\begin{array}{l}\text { - Students with Level } 1 \\
\text { PELA grade were twice } \\
\text { (adjusted odds ratio (AOR): } \\
1.95,95 \% \text { confidence } \\
\text { interval }(\mathrm{Cl}): 1.63-2.34 \text { ) as } \\
\text { probable to achieve a high } \\
\text { overall mark (>63\%) } \\
\text { - PELA (Level } 1 \text { ) was again } \\
\text { a significant and } \\
\text { independent predictor of } \\
\text { GPA (>4.2) }\end{array}$ \\
\hline $\begin{array}{l}\text { Koch et al. } \\
2011,611- \\
616\end{array}$ & $\begin{array}{l}\text { University } \\
\text { in western } \\
\text { Sydney, } \\
\text { Australia }\end{array}$ & $\begin{array}{l}2009 \text { single cohort } \\
\text { of } 62 \text { graduate- } \\
\text { entry nursing } \\
\text { students } 6 \text { months } \\
\text { post course } \\
\text { commencement } \\
\text { (mean age } 26.5 \\
\text { years; } 90 \% \text { female } \\
\text { and } 10 \% \text { male) }\end{array}$ & $\begin{array}{l}\text { Prospective } \\
\text { survey }\end{array}$ & $\begin{array}{l}\text { - ELAS } \\
\text { - Perceived } \\
\text { Academic Control } \\
\text { (PAC): } 8 \text { items } \\
\text { related to influencing } \\
\text { academic } \\
\text { achievement } \\
\text { outcomes } \\
\text { - VARK (visual, } \\
\text { aural/auditory, } \\
\text { read/write, and } \\
\text { kinaesthetic): } 16 \\
\text { items used to assess } \\
\text { learning preferences }\end{array}$ & $\begin{array}{l}\text { - Kinesthetic sensory }(\beta= \\
0.39, p=0.009) \\
\text { - Age, international } \\
\text { students, perceived } \\
\text { academic control, ELAS } \\
\text { and VARK score: } \\
\text { Kinaesthetic revealed } \\
\text { adjusted } \mathrm{R}^{2} \text { of } 0.17 .\end{array}$ \\
\hline
\end{tabular}




\begin{tabular}{|c|c|c|c|c|c|}
\hline Author (year) & $\begin{array}{l}\text { Setting/ } \\
\text { location }\end{array}$ & Participants & $\begin{array}{c}\text { Study } \\
\text { design }\end{array}$ & Instrument(s) used & $\begin{array}{c}\text { Significant predictors of } \\
\text { performance }\end{array}$ \\
\hline $\begin{array}{l}\text { Lancia et al. } \\
2013,1501- \\
1505\end{array}$ & Italy & $\begin{array}{l}\text { Total of } 1006 \text { from } \\
5 \text { cohorts of } \\
\text { nursing students, } \\
\text { matriculated in } \\
\text { consecutive } \\
\text { academic years } \\
\text { from } 2004 \text { to } 2008 \\
\text { (mean age } 23.36 \\
\text { years) }\end{array}$ & $\begin{array}{l}\text { Retrospective } \\
\text { observational }\end{array}$ & $\begin{array}{l}\text { Survey details not } \\
\text { clear. }\end{array}$ & $\begin{array}{l}61.2 \% \text { concluded their } \\
\text { degree within the legal } \\
\text { duration allowed. } \\
\text { - Age }(\beta=0.160, p=0.000) \\
\text { - Gender, age, upper- } \\
\text { secondary school attended } \\
\text { and grade of upper- } \\
\text { secondary diploma } \\
\text { revealed adjusted } \mathrm{R}^{2} \text { of } \\
0.27\end{array}$ \\
\hline $\begin{array}{l}\text { Pitt et al. } \\
2014,866- \\
871\end{array}$ & Australia & $\begin{array}{l}138 \text { preregistration } \\
\text { nursing students } \\
\text { (mean age } 27 \\
\text { years; } 86 \% \text { female } \\
\text { and } 14 \% \text { male) }\end{array}$ & $\begin{array}{l}\text { Longitudinal } \\
\text { descriptive } \\
\text { correlational } \\
\text { study }\end{array}$ & $\begin{array}{l}\text { - Personal Qualities } \\
\text { Assessment (PQA): } \\
\text { designed to explore } \\
\text { a broad range of } \\
\text { personal qualities in } \\
\text { healthcare } \\
\text { professionals } \\
\text { - Narcissism, } \\
\text { Aloofness, } \\
\text { Confidence, Empathy } \\
\text { (NACE) scale } \\
\text { - Self-Appraisal } \\
\text { Inventory (SAI) }\end{array}$ & $\begin{array}{l}\text { Clinical performance } \\
- \text { As the (self) confidence } \\
\text { score rose by } 1 \text {, the chance } \\
\text { of being assessed as } \\
\text { competent rose by } 20 \% \\
\text { - As the score of self- } \\
\text { control rose by } 1 \text {, the } \\
\text { chance of being assessed } \\
\text { as competent rose by } 6 \% \text {. } \\
\text { Progression } \\
- \text { As the resilience score } \\
\text { rose by } 1 \text {, the chance of } \\
\text { completing the programme } \\
\text { study rose by } 3 \% \text {. }\end{array}$ \\
\hline $\begin{array}{l}\text { Salamonson } \\
\text { et al. } 2014, \\
127-131\end{array}$ & Australia & $\begin{array}{l}\text { Total of } 352 \\
\text { participants } \\
\text { followed up over } 6 \text { - } \\
\text { year period from } \\
\text { beginning } 2004 \text { to } \\
\text { end } 2009 \text { (mean } \\
\text { age } 25.7 \text { years; } \\
84 \% \text { female and } \\
16 \% \text { male) }\end{array}$ & $\begin{array}{l}\text { Longitudinal, } \\
\text { cohort }\end{array}$ & $\begin{array}{l}\text { - Survey: contained } \\
\text { items related to } \\
\text { students' socio- } \\
\text { demographic } \\
\text { characteristics, } \\
\text { including } \\
\text { engagement in paid } \\
\text { employment during } \\
\text { semester }\end{array}$ & $\begin{array}{l}\text { - Students who selected } \\
\text { nursing as their first choice } \\
\text { for study were virtually } \\
\text { twice as likely (odds ratio } \\
\text { (OR): } 1.99,95 \% \mathrm{Cl}: 1.07- \\
3.68) \text { to complete their } \\
\text { nursing programme than } \\
\text { those who did not. } \\
\text { - Male students (OR: } 1.93 \text {, } \\
95 \% \mathrm{Cl}: 1.07-3.46) \text { and } \\
\text { those who worked more } \\
\text { than } 16 \text { hours per week } \\
\text { (OR: } 1.80,95 \% \mathrm{Cl}: 1.09- \\
2.99) \text { were less likely to } \\
\text { complete their nursing } \\
\text { programme than their } \\
\text { counterparts. }\end{array}$ \\
\hline $\begin{array}{l}\text { Ali and Naylor } \\
2010,157- \\
162\end{array}$ & Pakistan & $\begin{array}{l}\text { First-year } 544 ; \\
\text { second-year } 500 ; \\
\text { third-year } 343 \text { (age } \\
\text { range } 15-34 \text { years } \\
\text { on admission to } \\
\text { the nursing } \\
\text { programme; } 72.9 \% \\
\text { female and } 27.1 \% \\
\text { male) }\end{array}$ & $\begin{array}{l}\text { Exploratory } \\
\text { co-relational }\end{array}$ & $\begin{array}{l}\text { Data were collected } \\
\text { by reviewing student } \\
\text { records. Information } \\
\text { on enrolment } \\
\text { characteristics was } \\
\text { collected from } \\
\text { students' files in their } \\
\text { respective } \\
\text { institutions. }\end{array}$ & $\begin{array}{l}\text { Year one: Pre-admission } \\
\text { qualification, previous } \\
\text { academic performance, and } \\
\text { school type accounted for } \\
36.6 \%\left(\mathrm{R}^{2}=0.366\right) \text { of } \\
\text { variances in the } \\
\text { examination grade. } \\
\text { Year two: Pre-admission } \\
\text { qualification, previous } \\
\text { academic performance, } \\
\text { type of school, and gender } \\
\text { explained } 44.3 \%\left(\mathrm{R}^{2}=\right. \\
0.443) \text { of variability in the } \\
\text { examination grade } \\
\text { distributions. } \\
\text { Year three: Pre-admission } \\
\text { qualification, previous } \\
\text { academic performance, } \\
\text { academic performance in } \\
\text { year one and year two, } \\
\text { gender, and place of } \\
\text { domicile accounted for } \\
50.4 \%\left(R^{2}=0.504\right) \text { of } \\
\text { variability in the } \\
\text { examination grade } \\
\text { distributions. }\end{array}$ \\
\hline
\end{tabular}




\begin{tabular}{|c|c|c|c|c|c|}
\hline Author (year) & $\begin{array}{l}\text { Setting/ } \\
\text { location }\end{array}$ & Participants & $\begin{array}{l}\text { Study } \\
\text { design }\end{array}$ & Instrument(s) used & $\begin{array}{l}\text { Significant predictors of } \\
\text { performance }\end{array}$ \\
\hline $\begin{array}{l}\text { Timer and } \\
\text { Clauson } \\
2011,601- \\
606\end{array}$ & $\begin{array}{l}\text { Canadian } \\
\text { university, } \\
\text { Canada }\end{array}$ & $\begin{array}{l}\text { Total of } 249 \\
\text { baccalaureate } \\
\text { nursing students } \\
\text { over a four-year } \\
\text { study period } \\
\text { (2002-2006) } \\
\text { (mean age } 27.3 \\
\text { years on } \\
\text { admission; } 86.3 \% \\
\text { female and } 13.7 \% \\
\text { male) }\end{array}$ & $\begin{array}{l}\text { Retrospective } \\
\text { correlational } \\
\text { study of the } \\
\text { predictive } \\
\text { utility }\end{array}$ & $\begin{array}{l}\text { - Supplemental } \\
\text { application score: } \\
\text { measures applicant's } \\
\text { leadership capacity, } \\
\text { ability to work with } \\
\text { others, diversity of } \\
\text { experience, and } \\
\text { suitability for nursing } \\
\text { - Interview score: } \\
\text { content of interviews } \\
\text { included } \\
\text { assessments of } \\
\text { motivation, nursing } \\
\text { awareness, and } \\
\text { problem solving }\end{array}$ & $\begin{array}{l}\text { Grade for six nursing } \\
\text { courses } \\
- \text { Gender (male) }(\beta=- \\
0.13) \text {, age }(\beta=-0.26) \text {, } \\
\text { ethnic minority status ( } \beta=- \\
0.22), \text { and admission GPA } \\
(\beta=0.36) \text { were significant } \\
\text { predictor variables. } \\
\left.\text { - The model (adj } R^{2}=0.26\right) \\
\text { was statistically significant } \\
\text { ( } p<0.001) \text {. } \\
\text { Final GPA at graduation } \\
- \text { Age }(\beta=-0.30), \text { ethnic } \\
\text { minority status }(\beta=-0.24) \text {, } \\
\text { admission GPA }(\beta=0.36) \\
\text { and supplemental score }(\beta \\
=0.13) \text { were significant } \\
\text { predictors. } \\
\left.- \text { The model (adj } R^{2}=0.36\right) \\
\text { was statistically significant } \\
(p<0.001) \text {. }\end{array}$ \\
\hline $\begin{array}{l}\text { Wambuguh, } \\
\text { Eckfield and } \\
\text { Van } \\
\text { Hofwegen } \\
2016, \\
10.1515 \text { /ijnes- } \\
2015-0088\end{array}$ & $\begin{array}{l}\text { San } \\
\text { Francisco } \\
\text { Bay, USA }\end{array}$ & $\begin{array}{l}513 \text { final-year } \\
\text { nursing students } \\
\text { from 2009-2014. }\end{array}$ & Not clear & $\begin{array}{l}\text { - Test of Essential } \\
\text { Academic Skills } \\
\text { (TEAS). A } \\
\text { standardized, } \\
\text { multiple-choice exam } \\
\text { for prospective } \\
\text { nursing in the USA. }\end{array}$ & $\begin{array}{l}\text { Graduation } \\
\text { - Students with TEAS score } \\
\geq 82 \text { increased their odds of } \\
\text { graduation by a factor of } \\
2.14(p=0.01 \text { ). } \\
\text { Nursing programme GPA } \\
\text { - Students with TEAS score } \\
\text { of at least } 82 \text { have a } 60 \% \\
\text { probability of attaining a } \\
\text { GPA of } 3.25 \text {. Likewise, } \\
\text { TEAS scores }<82 \text { give a } \\
\text { probability of } 47 \% \text { (lower by } \\
13 \% \text { ). } \\
- \text { Student with a pre- } \\
\text { admission science GPA } \\
\text { score of at least } 3.8 \text { has a } \\
61 \% \text { probability of attaining } \\
\text { a graduation GPA of } 3.25 \text {. } \\
\text { Similarly, pre-admission } \\
\text { science GPA <3.8 gives a } \\
\text { probability of } 47 \% \text { (lower by } \\
14 \% \text { ). }\end{array}$ \\
\hline $\begin{array}{l}\text { Kowitlawakul, } \\
\text { Brenkus and } \\
\text { Dugan 2013, } \\
38-43\end{array}$ & & $\begin{array}{l}56 \text { female and } 4 \\
\text { male students } \\
\text { enrolled for first- } \\
\text { semester, second- } \\
\text { degree Bachelor of } \\
\text { Science in Nursing }\end{array}$ & Descriptive & $\begin{array}{l}\text { Pre-existing data } \\
\text { from academic } \\
\text { records were used. }\end{array}$ & $\begin{array}{l}\text { - Positive correlations } \\
\text { between first semester } \\
\text { GPAs and previous degree } \\
\text { GPAs }(r=0.348, p<0.005) \text {, } \\
\text { prerequisite GPAs }(r= \\
0.380, p<0.05) \text { and } \\
\text { admissions test scores ( } r= \\
0.678, p<0.05) \text {. } \\
\text { - Previous GPAs ( } \beta= \\
0.242) \text { and admissions tests } \\
\text { scores ( } \beta=0.612 \text { ) were } \\
\text { significant predictors ( } p= \\
0.01) . \text { Previous degree } \\
\text { GPAs, prerequisite GPAs, } \\
\text { and admissions test scores } \\
\text { revealed } R^{2} \text { of } 0.54 \text {. }\end{array}$ \\
\hline $\begin{array}{l}\text { Abele, } \\
\text { Penprase and } \\
\text { Ternes 2013, } \\
\text { 258-261 }\end{array}$ & USA & $\begin{array}{l}327 \text { students } \\
\text { placed on } \\
\text { probation or } \\
\text { dismissed from a } \\
\text { Midwest school of } \\
\text { nursing between } \\
2002 \text { and } 2010 \\
\text { (mean age } 27.2\end{array}$ & $\begin{array}{l}\text { Retrospective } \\
\text { review of an } \\
\text { existing } \\
\text { student } \\
\text { database }\end{array}$ & $\begin{array}{l}\text { Review of an existing } \\
\text { student database: } \\
\text { Student records } \\
\text { provided data. }\end{array}$ & $\begin{array}{l}\text { - PSY } 225 \text { ( } \beta=0.47 \text {, } \\
p<0.05) \text { as well as the total } \\
\text { number of courses failed ( } \beta \\
=-1.03, p<0.001) \text { had a } \\
\text { significant impact in } \\
\text { determining whether or not } \\
\text { probationary students } \\
\text { eventually completed the }\end{array}$ \\
\hline
\end{tabular}




\begin{tabular}{|c|c|c|c|c|c|}
\hline Author (year) & $\begin{array}{l}\text { Setting/ } \\
\text { location }\end{array}$ & Participants & $\begin{array}{l}\text { Study } \\
\text { design }\end{array}$ & Instrument(s) used & $\begin{array}{c}\text { Significant predictors of } \\
\text { performance }\end{array}$ \\
\hline & & $\begin{array}{l}\text { years; } 249 \text { female } \\
\text { and } 78 \text { male) }\end{array}$ & & & programme. \\
\hline $\begin{array}{l}\text { Fernandez, } \\
\text { Salamonson } \\
\text { and Griffiths } \\
2012,3485- \\
3492\end{array}$ & $\begin{array}{l}\text { Australian } \\
\text { university }\end{array}$ & $\begin{array}{l}81 \text { first-year } \\
\text { nursing students, a } \\
\text { cohort from } 2010 \\
\text { (mean age } 29.0 \\
\text { years; } 80 \% \text { female } \\
\text { and } 20 \% \text { male) }\end{array}$ & $\begin{array}{l}\text { Prospective } \\
\text { survey }\end{array}$ & $\begin{array}{l}\text { - Trait Emotional } \\
\text { Intelligence } \\
\text { Questionnaire-Short } \\
\text { Form (TEIQue-SF) } \\
\text { - Motivated } \\
\text { Strategies for } \\
\text { Learning } \\
\text { Questionnaire } \\
\text { (MSLQ) }\end{array}$ & $\begin{array}{l}\text { - Trait EI (TEIQue-SF) }(\beta= \\
0.25, p=0.023) \\
\text { - MSLQ (extrinsic goal } \\
\text { orientation) had a negative } \\
\text { impact on academic } \\
\text { performance }(\beta=-0.23, p= \\
0.035) \text {. }\end{array}$ \\
\hline Goff 2011, 1 & USA & $\begin{array}{l}53 \text { BN students } \\
\text { (mean age } 24.8 \\
\text { years; } 92.5 \% \\
\text { female; } 84.9 \% \\
\text { Caucasian) }\end{array}$ & $\begin{array}{l}\text { Explanatory } \\
\text { correlational } \\
\text { study }\end{array}$ & $\begin{array}{l}\text { - Student-life Stress } \\
\text { Inventory (SSI); used } \\
\text { to measure college } \\
\text { student stress }\end{array}$ & $\begin{array}{l}\text { - Age was a significant } \\
\text { predictor }(\beta=0.417, \\
p<0.01) \text { and accounted for } \\
16 \%\left(R^{2}=0.16\right) \text { of } \\
\text { variances in the GPA } \\
\text { distributions. }\end{array}$ \\
\hline
\end{tabular}

\section{Overview of the reviewed studies}

The final sample of 17 studies included in the systematic review consisted of 5 prospective survey studies (Salamonson, Andrew and Everett 2009, 123-132; Salamonson and Andrew 2006, 342-349; Glew et al. 2015, 1142-1147; Koch et al. 2011, 611-616; Fernandez, Salamonson and Griffiths 2012, 3485-3492); 6 studies with an explanatory and correlational component (Goff 2011, 1; Ali and Naylor 2010, 157-162; Cesario, Cesario and Cesario 2013, 141-146; Kowitlawakul, Brenkus and Dugan 2013, 38-43; Pitt et al. 2014, 866-871; Timer and Clauson 2011, 601-606); 2 prospective correlational studies (Wan Chik et al. 2012, 387393; Everett et al. 2013, 709-713); 1 study with a retrospective observational design (Lancia et al. 2013, 1501-1505); 1 longitudinal, cohort study (Salamonson et al. 2014, 127-131); and 1 retrospective review of an existing database (Abele, Penprase and Ternes 2013, 258-261). One study (Wambuguh, Eckfield and Van Hofwegen 2016, 10.1515/ijnes-2015-0088) did not completely describe the designs that were used.

The geographical locations of the studies were as follows: 8 studies in Australia (Salamonson and Andrew 2006, 342-349; Salamonson, Andrew and Everett 2009, 123-132; Koch et al. 2011, 611-616; Fernandez, Salamonson and Griffiths 2012, 3485-3492; Everett et al. 2013, 709-713; Pitt et al. 2014, 866-871; Salamonson et al. 2014, 127-131; Glew et al. 2015, 1142-1147), 4 studies in the USA (Goff 2011, 1; Abele, Penprase and Ternes 2013, 258261; Cesario, Cesario and Cesario 2013, 141-146; Wambuguh, Eckfield and Van Hofwegen 2016, 10.1515/ijnes-2015-0088), 1 study in Canada (Timer and Clauson 2011, 601-606), 1 study in Italy (Lancia et al. 2013, 1501-1505), 1 study in Malaysia (Wan Chik et al. 2012, 387393) and 1 study in Pakistan (Ali and Naylor 2010, 157-162). Of all the studies included in the review, geographic location was not specified for 1 study (Kowitlawakul, Brenkus and Dugan 2013, 38-43). Participants in the studies were all nursing students enrolled in various 
undergraduate nursing programmes at their respective institutions.

\section{Measures of academic performance and success}

A number of outcome variables were used to assess academic performance and success amongst nursing students. Furthermore, the results revealed that 5 of the shortlisted studies had 2 or more outcome variables. Eleven studies addressed academic performance as grade point average (GPA) at various levels of undergraduate programmes (Cesario, Cesario and Cesario 2013, 141-146; Wan Chik et al. 2012, 387-393; Everett et al. 2013, 709-713; Glew et al. 2015, 11421147; Koch et al. 2011, 611-616; Pitt et al. 2014, 866-871; Timer and Clauson 2011, 601-606; Wambuguh, Eckfield and Van Hofwegen 2016, 10.1515/ijnes-2015-0088; Kowitlawakul, Brenkus and Dugan 2013, 38-43; Fernandez, Salamonson and Griffiths 2012, 3485-3492; Goff 2011, 1), 2 studies addressed academic performance as the score obtained by nursing students in pathophysiology (Salamonson and Andrew 2006, 342-349; Salamonson, Andrew and Everett 2009, 123-132), 2 studies addressed academic performance as the score obtained in clinical practice modules (Salamonson and Andrew 2006, 342-349; (Pitt et al. 2014, 866-871), 1 study addressed academic performance as grades in nursing theory (Pitt et al. 2014, 866-871), and 1 study addressed academic performance as grades awarded by nursing examination boards from respective countries (Ali and Naylor 2010, 157-162).

Of the 17 studies included in the present systematic review, 6 studies addressed academic success (promotion to next level of study or completing the programme) or failure/attrition amongst various levels of undergraduate nursing programmes (Cesario, Cesario and Cesario 2013, 141-146; Lancia et al. 2013, 1501-1505; Abele, Penprase and Ternes 2013, 258-261; Salamonson et al. 2014, 127-131; Pitt et al. 2014, 866-871; Wambuguh, Eckfield and Van Hofwegen 2016, 10.1515/ijnes-2015-0088).

\section{Factors that predict academic performance and success}

To provide assistance with analysis across all 17 shortlisted studies in this review, the factors that predict academic performance and success were categorized into 6 groups: (1) student profile characteristics; (2) student affective factors; (3) academic factors; (4) environmental factors (5) academic outcomes; and (6) psychological outcomes. This categorization was presented by Jeffreys (2015) and is based on the Nursing Universal Retention and Success (NURS) model.

\section{Student profile characteristics}

Age, gender and language proficiency were the most consistent student profile characteristics 
that displayed significant predictive powers for academic performance and success. Female and older students were found to be more associated with higher academic performance and academic success, and their male and younger counterparts were associated with unsatisfactory academic performance and a high attrition rate. Selection of nursing as first choice for study was also consistent in determining academic performance and success of UNs. In contrast, ethnicity as a characteristic of nursing students revealed inconsistent findings.

Age was a significant predictor of academic performance and success (Salamonson and Andrew 2006, 342-349; Everett et al. 2013, 709-713; Lancia et al. 2013, 1501-1505; Timer and Clauson 2011, 601-606; Goff 2011, 1). The results indicated that the older the student, the higher the probability of achieving the desired outcome.

Gender was identified as a significant predictor of academic performance and success (Wan Chik et al. 2012, 387-393; Salamonson et al. 2014, 127-131; Ali and Naylor 2010, 157162; Timer and Clauson 2011, 601-606). Female nursing students were more likely to outperform their male counterparts.

Language proficiency was tested in various ways for different studies (English-speaking at home, ELAS and PELA) and found to be a significant predictor of academic performance (Salamonson and Andrew 2006, 342-349; Everett et al. 2013, 709-713; Glew et al. 2015, 11421147). Low English language proficiency was associated with unsatisfactory academic performance. Timer and Clauson (2011, 601-606) found ethnic minority status to be a negative $(\beta=-0.24)$ and significant predictor of academic performance.

Only one study tested the predictive validity of selecting nursing as first study choice on academic success (Salamonson et al. 2014, 127-131), and revealed that students who selected nursing as their first study choice were nearly twice as likely (OR: $1.9995 \%$ CI: 1.07-3.68) to complete their nursing programme as those who did not.

Four studies shortlisted for the present systematic review indicated that prior educational experiences are a significant predictor of academic performance, success and retention of nursing student (Ali and Naylor 2010, 157-162; Timer and Clauson 2011, 601-606; Wambuguh, Eckfield and Van Hofwegen 2016, 10.1515/ijnes-2015-0088; Kowitlawakul, Brenkus and Dugan 2013, 38-43). Prior educational experiences were denoted in various ways including pre-admission qualifications, admission GPA, supplemental application score and pre-admission science GPA. Ali and Naylor (2010, 157-162) reported that pre-admission qualifications as well as previous academic performance (measured by the percentages obtained in previous programmes) had a significant influence on academic performance. Admission GPA $(\beta=0.36)$, and supplemental application score $(\beta=0.13)$ were also found to be significant predictor variables of nursing students' performance and success (Timer and Clauson 2011, 
601-606). Likewise, a student with a pre-admission science GPA score of at least 3.8 had a 61 per cent probability of attaining a graduation GPA of 3.25 (Wambuguh, Eckfield and Van Hofwegen 2016, 10.1515/ijnes-2015-0088). Kowitlawakul, Brenkus and Dugan (2013, 38-43) indicated that previous GPAs $(\beta=0.242, p=0.01)$, admission tests scores $(\beta=0.612, p=0.00)$ and prerequisite GPAs accounted for 54 per cent $\left(\mathrm{R}^{2}=0.54\right)$ of the variances in the Bachelor of Nursing $(\mathrm{BN})$ first semester GPA distributions.

\section{Student affective factors}

Of the 17 studies shortlisted for this review, only 3 tested the components of student affective factors. Pitt et al. (2014, 866-871) reported that aloofness and confidence (measured by the Narcissism, Aloofness, Confidence, Empathy (NACE) scale) had a significant impact on nursing students' academic performance. They found that aloofness score was negatively correlated $(p<0.05$ to $p<0.01)$ with academic performance. In contrast, confidence scores $(p<0.05)$ were positively correlated with academic performance. Self-control and resilience (measured by the Self-Appraisal Inventory (SAI)) were reported to be positive and significant predictors $(p<0.05$ to $p<0.01)$ of academic performance and success (Pitt et al. 2014, 866-871).

Fernandez, Salamonson and Griffiths (2012, 3485-3492) found that emotional intelligence (Trait Emotional Intelligence Questionnaire-Short Form (TEIQue-SF)) and motivation (Motivated Strategies for Learning Questionnaire (MSLQ)) were significant predictors of academic performance. The results indicated positive and significant relationships $(\beta=0.25, p=0.023)$ between emotional intelligence and academic performance. The results also indicated that extrinsic goal orientation had an inverse impact $(\beta=-0.23, p=0.035)$ on academic performance.

Partaking in organised music programmes and number of years taking music lessons were identified as positive and significant predictors of graduating (Cesario, Cesario and Cesario 2013, 141-146). They explained 66 per cent (Nagelkerke $\mathrm{R}^{2}=0.66, p<0.001$ ) of variances in completing the nursing programme distributions.

\section{Academic factors}

Homework completion, lecture attendance, kinaesthetic learning preference and Test of Essential Academic Skills (TEAS) were found to have a significant impact on the academic performance and success of nursing students. Salamonson, Andrew and Everett (2009, 123132) reported that homework completion $(>50 \%)(\beta=0.44, p<0.001)$ and lecture attendance $(>80 \%)(\beta=0.21, p=0.011)$ were positive and significant predictors of performance in pathophysiology subjects. 
A study by Koch et al. (2011, 611-616) testing the predictive power of visual, aural/auditory, read/write and kinaesthetic (VARK) learning preferences indicated that kinaesthetic sensory mode was a positive and significant predictor $(\beta=0.39, p=0.009)$ of academic performance at the 6-month follow-up in graduate entry nursing. Kinaesthetic sensory mode accounted for 17 per cent $\left(\mathrm{R}^{2}=0.17\right)$ of variability in the first semester GPA distribution.

TEAS was identified to be a statistically significant $(p=0.01)$ and positive predictor of graduating and high nursing programme GPA (Wambuguh, Eckfield and Van Hofwegen 2016, 10.1515/ijnes-2015-0088). Wambuguh, Eckfield and Van Hofwegen (2016, 10.1515/ijnes2015-0088) found that a TEAS score $\geq 82$ increased the odds of graduation by a factor of 2.14. Furthermore, a student with a TEAS score of at least 82 had a 60 per cent likelihood of attaining a GPA of 3.25 .

\section{Environmental factors}

Two shortlisted studies by Salamonson, Andrew and Everett (2009, 123-132) and Everett et al. (2013, 709-713) reported that hours of part-time employment ( $\geq 16$ hours/week) had a significant and negative impact $(p \leq 0.001)$ on academic performance of nursing students. Students engaged in >16 hours/week of employment (OR 1.80 95\% CI: 1.09-2.99) were less likely to complete their nursing programme than were their counterparts (Salamonson et al. 2014, 127-131). Place of domicile (whether rural or urban) as well as type of nursing school (public or private) were found to be significantly associated with academic success (Ali and Naylor 2010, 157-162).

\section{Academic outcomes}

Academic outcome in precious levels has displayed consistent results. Academic performance in year one as well as academic performance in year two were found to be significant predictors of academic performance and success of nursing students (Ali and Naylor 2010, 157-162). Abele, Penprase and Ternes (2013, 258-261) conducted a study aiming to identify undergraduate courses that serve as predictors of success for nursing students completing a BSN programme. The findings for this study revealed that not only background in science predicts performance in nursing but also that psychology modules may hold predictive powers. Abele, Penprase and Ternes $(2013,258-261)$ reported that PSY $225(\beta=0.47, p<0.05)$ was a positive and significant predictor of completing a nursing programme.

\section{Psychological outcomes}

Of the 17 shortlisted studies, only one tested the effect of psychological outcomes on nursing 
student performance (Goff 2011, 1). This study examined the link between learned resourcefulness, stressors and academic performance in baccalaureate nursing students using the Student-life Stress Inventory (SSI). The results revealed that there was no significant relationship between personal stress levels and academic stress levels on the academic performance of nursing students (Goff 2011, 1).

\section{DISCUSSION}

The aim of this study was to determine the predictors of academic performance among undergraduate nursing students. The findings from the present study suggest potential factors that might have significant predictive validity on academic performance, success and, ultimately, retention of UNs. Jeffreys (2015) suggests that retention, success and optimal performance of UNs is a result of interaction between student profile characteristics, student affective factors, academic factors, environmental factors, academic outcomes, psychological outcomes, outside surrounding factors as well as the professional integration factors.

Jeffreys (2015) describes student profile characteristics as the qualities of the students prior to commencing the undergraduate programme. According to the NURS model by Jeffreys (2015), student profile characteristics are attributes of the students prior to entering the nursing programme. Furthermore, Jeffreys (2015) proposes that being aware of the student profile characteristics can assist nurse educators in developing proactive and ongoing strategies to capitalize on student strength and improve weakness. It has been demonstrated in the literature that student profile characteristics such as age, gender, language proficiency, ethnicity, educational experience and programme choice were significant predictors of academic performance and success amongst nursing students (Ali and Naylor 2010, 157-162; Oducado and Penuela 2014, 21-28; Mthimunye, Daniels and Pedro 2015); Alshammari et al. 2017, 60). The present review revealed parallel findings by identifying older students, female gender, English language proficiency, majority ethnic status, pre-admission qualifications, high admission GPA, high supplemental application score, high pre-admission science GPA and selecting nursing as first choice for study, as significant predictors of academic performance. In contrast, a surprising finding from a study conducted by Mthimunye, Daniels and Pedro (2015) in SA to determine the predictive power of ethnicity in predicting academic performance amongst nursing students reported that ethnic minority status was a positive and significant predictor of academic performance (Kruskal-Wallis, $p<0.05$ ). Given the racial and ethnic history as well as current racial and ethnic inequalities in SA, it might be expected that students belonging to a privileged ethnic group would have more advantage than their less privileged counterparts. This finding highlights that ethnicity as a predictor of academic performance may 
depend on the context.

According to Jeffreys (2015) students' affective factors are factors related to students' attitudes, motivation, self-efficacy, cultural values and beliefs (CVB) about learning. CVB in the NURS conceptual model acknowledges that all nursing students enroll in nursing programmes with values and beliefs that directly or indirectly influence their thought process, decision making as well as their actions throughout their student life and in all dimensions of the nursing profession. Furthermore, the NURS model a recognizes that conflict between CVB, nursing profession, academic environment and nursing education may contribute towards an increase in nursing students' stress levels and ultimately lead to unsatisfactory academic performance, lack of motivation and attrition. The literature validated that students' affective factors such as motivation and self-efficacy have a significant impact on their academic performance (McLaughlin, Moutray and Muldoon 2008, 211-221). The present study demonstrated that affective factors such as emotional intelligence, self-control and resilience were predictors of satisfactory academic performance. In contrast, this study indicated that students who are extrinsic goal orientated are at risk of unsatisfactory academic performance and ultimately dropping out of nursing programmes. Extrinsic goal orientated students have a tendency to focus on achieving higher grades and obtaining rewards. Although this type of motivation may not appear to be absolutely negative, it may have inverse effects in nursing programmes owing to its clinical nature. A possible explanation for this finding might be that nursing as a profession poses various challenges to students and thus requires a high level of perseverance to overcome challenges.

An interesting finding in this category was the impact that organised music programmes and number of years taking music lessons had on the performance of nursing students. Previous research has emphasised the impact of music on the cognitive development of children and adults with cognitive disabilities (Moreno et al. 2011, 1425-1433). This finding should be interpreted with caution as there is marginal scientific evidence to back it up. A possible account for this finding could be that extended exposure to music might improve listening skills and therefore be advantageous for partaking students.

The NURS model proposes that external environmental factors to the academic process such as financial support, family emotional support, family responsibilities, childcare arrangements, employment hours, employment responsibilities, transport arrangements and living arrangements have a significant influence on the academic performance, success and retention of nursing students. Employment hours has been shown to be a consistent predictor of academic performance (Salamonson and Andrew 2006, 342-349; Salamonson, Andrew and Everett 2009, 123-132; Everett et al. 2013, 709-713; Salamonson et al. 2014, 127-131; Reyes 
et al. 2012, 218-221; Beerkens, Mägi and Lill 2011, 679-692) reported that in Colombia, Australia, USA and Europe approximately 50.3 per cent, 50 per cent, 49 per cent and 47 per cent of nursing students engage is some form of employment respectively. This percentages could be drastically high in developing counties such as South Africa given the poor socioeconomic status. The result of this review reported that hours of part-time employment hours has a negative impact $(p \leq 0.001)$ on academic performance of nursing students. The possible explanation for this finding could be the fact that the more hours spent in employment and employment responsibilities, the less time spent engaging with academic responsibilities.

Place of domicile (whether rural or urban) was found to be significantly associated with academic success (Ali and Naylor 2010, 157-162). In the contrary, a study conducted by Oducado and Penuela $(2014,21-28)$ in the Philippines aiming to discover and identify weather or not the students' academic and non-academic factors predict academic performance of nursing students found that the place of residence to is not a significant predictor of academic performance. This finding could be explained by the fact that students that are residing in urban areas may have easy access to a variety of services such as reliable internet connection as well as library services.

Somewhat expectedly, Ali and Naylor (2010, 157-162) found that students registered at private nursing schools outperformed their counterparts at public nursing schools. This finding could be explained by the fact that private nursing schools ordinarily provide various learning opportunities and exceptional facilities, more so than do public schools.

The NURS model proposes that academic factors such as personal study skill (reading, writing, listening, note-taking, literature search, preparation for examination, time management, and clinical judgment), study hours, class attendance, class schedule / timetable arrangements, and general academic services (such as library services, counseling services, electronic-learning support services) are vital variables that have a significant impact on academic performance, successes and retention of nursing students (Jeffreys, 2015). In the current study, academic engagement (homework completion and lecture attendance), academic disengagement (engaging in part-time employment during the study course) and TEAS were found to be significant predictors of academic performance. These results provide supplementary evidence that the issues relating to academic engagement and disengagement as well as acquiring essential academic skills in higher academic institutions should be addressed.

Only one study shortlisted in the present review (Koch et al. 2011, 611-616), conducted in Australia with a total sample of $62 \mathrm{BN}$ students, identified that a kinaesthetic learning approach is preferred amongst BN students. The results imply that nursing students retain information best through practical sessions, case-based studies and computer simulations, 
which may be explained by the fact that nursing is a practical profession. However, owing to a small sample size and lack of recent literature, these findings should be interpreted and generalized beyond their setting with caution.

NURS model by Jeffreys (2015) suggests that academic outcomes (grades obtained by the student during the programme, cumulative GPA for nursing programme, and overall GPA) and psychological outcomes such as satisfaction and stress can have a positive or negative influence on the retention, performance, progression and success of nursing students. Consistent with the present review, the value of academic outcomes in predicting academic performance, success and retention of nursing students has been demonstrated in previous studies (Newton and Moore, 2009; Newton, Smith, Moore and Magnan, 2007; Mthimunye, Daniels and Pedro 2015). The present review reveals that the academic outcome of previous years is a significant predictor of performance in subsequent year levels of undergraduate nursing programmes. The explanation for this finding could be a high level of articulation between the year levels of the undergraduate nursing programme. Interestingly, Abele, Penprase and Ternes (2013, 258-261) revealed that not only outcome in science modules predicts performance in subsequent year levels but also that psychology modules may hold predictive value for nursing programme completion. Study findings indicated that a non-nursing and non-science module can also significantly predict academic success.

Moscaritolo (2009, 17-23) reported that nursing students experience high levels of stress and anxiety in undergraduate programmes. High levels of academic stress and anxiety may have a negative impact on the academic performance of nursing students and ultimately increase the attrition (Hughes 2005, 21-36). The results of this review revealed that stress levels and academic stress levels had no significant influence on the academic performance of nursing students (Goff 2011, 1). This result cannot be generalizable beyond the setting as the sample size $(\mathrm{N}=53)$ was too small.

The NURS model proposes that professional integration as well as outside surrounding factors also contribute towards the success and retention of nursing students. Jeffreys (2015) described professional integration factors as "factors that enhance student interaction with the social system of the college environment within the context of professional socialization and career development". These factors include peer mentoring, enrichment programmes, advisements and helpfulness. Outside surrounding factors however, is described as factors that are beyond the student's or educators control. For the present systematic review, none of the shortlisted studies addressed these categories. 


\section{Limitations}

The review was initiated as a part of a major $\mathrm{PhD}$ project entitled "An intervention towards the improvement of academic performance, success and retention among Bachelor of Nursing students at a higher education institution in the Western Cape". The present systematic review not only gathers, appraises and recapitulates data but also analyses it and interprets it within a conceptual model. This approach has allowed the researcher to explore the factors that predict academic performance and success of nursing students, identify gaps in the literature and highlight directions for future research. Owing to the complexity of the topic, academic performance and success has been measured differently in different parts of the world and therefore does not allow a meta-analysis of the data. Future studies could be strengthened by being more specific regarding the outcome variables of interest.

Another limitation was that none of the studies employed a randomized controlled trial (RCT) and controlled clinical trial (CCT) design. Therefore, none of the shortlisted studies had a "strong" research design. Future studies could be strengthened by searching and shortlisting research studies that employed RCT and CCT design.

\section{CONCLUSION AND RECOMMENDATIONS}

Identifying significant predictors of academic performance, success and retention of nursing student is of utmost importance. The results of this review can assist nursing schools in early identification of students who are at risk of unsatisfactory academic performance and ultimately failing to complete their nursing programme.

\section{Nursing education}

The results of this review suggest that male students, students with low English language proficiency and those who are younger in age should be given support throughout the nursing programme. Likewise, students with low admission scores and those who did not select nursing as first choice of study should be identified and monitored.

Assessments should be held to examine emotional intelligence, self-control, resilience, academic skills and motivational characteristics of students prior to, or at the beginning of, the programme to identify at-risk students and those who are extrinsically motivated, so that their progress can be monitored.

The present review highlights the importance of implementing innovative measures to improve academic engagement. It is therefore recommended that students be given tasks allowing them to interact with their schoolwork, and these tasks should be rewarded with marks to ensure compliance. It is also most important that nurse educators encourage learning 
approaches that promote academic engagement, such as classroom presentations, case-based learning, cellphone applications and role playing. Encouraging and monitoring class attendance may also assist in promoting academic engagement.

Lastly, it is of vital importance that the academic outcome of students at each and every year level be monitored closely to identify underperforming students. Once these students are identified, remedial action should be implemented as early as possible to ensure retention and low attrition.

\section{Implications for nursing programmes in South Africa}

The findings of this study may be important in terms of understanding the predictors of academic performance in a South African context. The implication of this results could result in a rise in throughput rate of nursing students, thus increasing the number of much needed BN graduates to alleviate the shortage of nursing workforce. Furthermore, addressing this these challenges will result in improvement in quality of nursing programmes offered in South Africa as government subsidies are dependent on the throughput of students.

\section{Future research}

Academic performance, success and retention of nursing students is a diverse topic that needs further investigation to verify findings and discover new factors that may predict performance. This systematic review reveals that there is a need for research focusing on the impact of professional integration factors, outside surrounding factors, psychological outcomes, learning approach and teaching strategies on the academic performance of nursing students. It would also be interesting for future research to confirm the influence of psychology modules as well as the type of nursing school (private/public) on the performance of nursing students.

\section{REFERENCES}

Abele, C., B. Penprase and R. Ternes. 2013. A closer look at academic probation and attrition: What courses are predictive of nursing student success? Nurse Education Today 33(3): 258-261.

Ali, Parveen Azam and Paul B. Naylor. 2010. Association between academic and non-academic variables and academic success of diploma nursing students in Pakistan. Nurse Education Today 30(2): 157-162.

Alshammari, Farhan, Reynita Saguban, Eddieson Pasay-an, Ahmed Altheban and Layla Al-Shammari. 2017. Factors affecting the academic performance of student nurses: A cross-sectional study. Journal of Nursing Education and Practice 8(1): 60.

Armijo-Olivo, Susan, Carla R. Stiles, Neil A. Hagen, Patricia D. Biondo and Greta G. Cummings. 2012. Assessment of study quality for systematic reviews: A comparison of the Cochrane Collaboration risk of bias tool and the Effective Public Health Practice Project quality assessment tool: Methodological research. Journal of Evaluation in Clinical Practice 18(1): 12-18.

Bassir, S. H., P. Sadr-Eshkevari, S. Amirikhorheh and N. Y. Karimbux. 2014. Problem-based learning in dental education: A systematic review of the literature. Journal of Dental Education 78(1): 98- 
109.

Beauvais, Audrey M., Julie G. Stewart, Susan DeNisco and John E. Beauvais. 2014. Factors related to academic success among nursing students: A descriptive correlational research study. Nurse Education Today 34(6): 918-923.

Beerkens, Maarja, Eve Mägi and Liis Lill. 2011. University studies as a side job: Causes and consequences of massive student employment in estonia. Higher Education 61(6): 679-692.

Buerhaus, P. I., D. I. Auerbach and D. O. Staiger. 2009. The recent surge in nurse employment: Causes and implications. Health Affairs (Project Hope) 28(4): w657-668.

Cesario, S. K., R. J. Cesario and A. R. Cesario. 2013. Organized music instruction as a predictor of nursing student success. Nurse Educator 38(4): 141-146.

Everett, Bronwyn, Yenna Salamonson, Suza Trajkovski and Ritin Fernandez. 2013. Demographic and academic-related differences between standard-entry and graduate-entry nursing students: A prospective correlational survey. Nurse Education Today 33(7): 709-713.

Fernandez, Ritin, Yenna Salamonson and Rhonda Griffiths. 2012. Emotional intelligence as a predictor of academic performance in first-year accelerated graduate entry nursing students. Journal of Clinical Nursing 21(23): 3485-3492.

Glew, Paul J., Sharon P. Hillege, Yenna Salamonson, Kathleen Dixon, Anthony Good and Lien Lombardo. 2015. Predictive validity of the post-enrolment English language assessment tool for commencing undergraduate nursing students. Nurse Education Today 35(12): 1142-1147.

Goff, Anne-Marie. 2011. Stressors, academic performance, and learned resourcefulness in baccalaureate nursing students. International Journal of Nursing Education Scholarship 8: 1.

Higgins, Julian P. T. and Sally Green. 2011. Cochrane handbook for systematic reviews of interventions. Vol. 4. Hoboken: John Wiley \& Sons.

Hughes, Brian M. 2005. Study, examinations, and stress: Blood pressure assessments in college students. Educational Review 57(1): 21-36.

Jeffreys, Marianne R. 2012. Nursing student retention: Understanding the process and making a difference. New York City: Springer.

Jeffreys, Marianne R. 2015. Jeffreys's Nursing Universal Retention and Success Model: Overview and action ideas for optimizing outcomes A-Z. Nurse Education Today 35(3): 425-431.

Koch, Jane, Yenna Salamonson, John X. Rolley and Patricia M. Davidson. 2011. Learning preference as a predictor of academic performance in first year accelerated graduate entry nursing students: A prospective follow-up study. Nurse Education Today 31(6)(8): 611-616.

Kowitlawakul, Y., R. Brenkus and N. Dugan. 2013. Predictors for success for first semester, seconddegree bachelor of science in nursing students. International Journal of Nursing Practice 19 Suppl. 1: 38-43.

Lancia, Loreto, Cristina Petrucci, Fabio Giorgi, Angelo Dante and Maria Grazia Cifone. 2013. Academic success or failure in nursing students: Results of a retrospective observational study. Nurse Education Today 33(12): 1501-1505.

McLaughlin, Katrina, Marianne Moutray and Orla T. Muldoon. 2008. The role of personality and selfefficacy in the selection and retention of successful nursing students: A longitudinal study. Journal of Advanced Nursing 61(2): 211-221.

McNelis, Angela M., Debra S. Wellman, Joyce Splann Krothe, Deborah D. Hrisomalos, Jennifer L. McElveen and Rebecca J. South. 2010. Revision and evaluation of the Indiana University School of Nursing baccalaureate admission process. Journal of Professional Nursing 26(3): 188-195.

Moreno, S., E. Bialystok, R. Barac, E. G. Schellenberg, N. J. Cepeda and T. Chau. 2011. Short-term music training enhances verbal intelligence and executive function. Psychological Science 22(11): $1425-1433$.

Moscaritolo, Linda M. 2009. Interventional strategies to decrease nursing student anxiety in the clinical learning environment. Journal of Nursing Education 48(1): 17-23. 
Mthimunye, K. D. T., F. M. Daniels and A. Pedro. 2015. Predictors of academic performance and throughput among second-year nursing students at a university in the Western Cape. Master of Nursing Education; Master's Dissertation, University of the Western Cape, Cape Town.

Newton, Sarah E. and Gary Moore. 2009. Use of aptitude to understand bachelor of science in nursing student attrition and readiness for the National Council Licensure Examination-Registered Nurse. Journal of Professional Nursing 25(5): 273-278.

Newton, Sarah E., Laureen H. Smith, Gary Moore and Morris Magnan. 2007. Predicting early academic achievement in a baccalaureate nursing program. Journal of Professional Nursing 23(3): 144-149.

Oducado, Ryan Michael F. and Ayesha C. Penuela. 2014. Predictors of academic performance in professional nursing courses in a private nursing school in kalibo, aklan, philippines. Asia Pacific Journal of Education, Arts, and Sciences 1(5): 21-28.

Pitt, Victoria, David Powis, Tracy Levett-Jones and Sharyn Hunter. 2014. The influence of personal qualities on performance and progression in a pre-registration nursing programme. Nurse Education Today 34(5): 866-871.

Puertas, E. Benjamín, Carlos Arósquipa and Daniela Gutiérrez. 2013. Factors that influence a career choice in primary care among medical students from high-, middle-, and low-income countries: A systematic review. Revista Panamericana De Salud Pública 34(5): 351-358.

Reyes, H., V. Hartin, C. Loftin, D. Davenport and V. Carter. 2012. The impact of employment on nursing students' academic performance. Nurse Educator 37(5) (Sep-Oct): 218-221.

Salamonson, Yenna and Sharon Andrew. 2006. Academic performance in nursing students: Influence of part-time employment, age and ethnicity. Journal of Advanced Nursing 55(3): 342-349.

Salamonson, Yenna, Sharon Andrew and Bronwyn Everett. 2009. Academic engagement and disengagement as predictors of performance in pathophysiology among nursing students. Contemporary Nurse: A Journal for the Australian Nursing Profession 32(1-2): 123-132.

Salamonson, Yenna, Bronwyn Everett, Melissa Cooper, Lien Lombardo, Roslyn Weaver and Patricia M. Davidson. 2014. Nursing as first choice predicts nursing program completion. Nurse Education Today 34(1): 127-131.

SANC see South African Nursing Council.

Schmidt, B. and B. MacWilliams. 2011. Admission criteria for undergraduate nursing programs: A systematic review. Nurse Educator 36(4): 171-174.

Smith, Virginia A. 2016. Nursing student attrition and implications for pre-admission advisement. Journal of Nursing Education 29(5): 215-218.

South African Nursing Council. 2017. South African Nursing Council statistics. http://www. sanc.co.za/stats.htm (Accessed 13 April 2017).

Thomas, H. 2003. Quality assessment tool for quantitative studies. Effective Public Health Practice Project. Toronto: McMaster University.

Timer, Jennifer E. and Marion I. Clauson. 2011. The use of selective admissions tools to predict students' success in an advanced standing baccalaureate nursing program. Nurse Education Today 31(6): 601-606.

Wambuguh, O., M. Eckfield and L. Van Hofwegen. 2016. Examining the importance of admissions criteria in predicting nursing program success. International Journal of Nursing Education Scholarship 13(1): 10.1515/ijnes,2015-0088.

Wan Chik, W. Z., Y. Salamonson, B. Everett, L. M. Ramjan, N. Attwood, R. Weaver, Z. Saad and P. M. Davidson. 2012. Gender difference in academic performance of nursing students in a Malaysian university college. International Nursing Review 59(3): 387-393.

World Health Organization. 2010. Increasing access to health workers in remote and rural areas through improved retention: Global policy recommendations. Geneva: World Health Organization. 